\title{
Slaying RAS with a synthetic lethal weapon
}

\author{
Archana Bommi-Reddy ${ }^{1}$, William G Kaelin Jr ${ }^{1,2}$ \\ ${ }^{1}$ Department of Medical Oncology, Dana-Farber Cancer Institute and Harvard Medical School, Boston, MA 02115, USA; ${ }^{2}$ How- \\ ard Hughes Institute, Chevy Chase, MD 20815, USA \\ Cell Research (2010) 20:119-121. doi:10.1038/cr.2010.16; published online 1 February 2010
}

Most chemotherapeutic agents in clinical use today are promiscuous, that is, they kill both normal and rapidly dividing cancer cells. Exploiting inherent differences between cancer and normal cells is fundamental to enhancing selectivity and minimizing the toxicity of anti-cancer drugs. Clearly, one way of obtaining an enhanced therapeutic index (defined as the dose at which toxic effects are observed divided by the dose at which therapeutic effects occur) would be to identify targets that are unique to cancer cells and not present in normal cells. A second way to enhance selectivity, however, would be to identify scenarios where the requirement for a target that is present in both normal and cancer cells has been quantitatively or qualitatively altered by virtue of the genetic (or epigenetic) changes that occurred during malignant transformation [1].

One possible strategy to achieve this selectivity would be to exploit synthetic lethal gene interactions when choosing amongst potential cancer drug targets. Two genes ('A' and ' $B$ ') are said to be 'synthetic lethal' if mutation in either gene alone is compatible with viability but concomitant mutation in both genes results in the demise of the cell $[2,3]$. Thus, if gene ' $A$ ' is mutated in a cancer cell then inhibiting its synthetic lethal interactor, gene 'B', should theoretically

Correspondence: William G Kaelin Jr

E-mail:William_Kaelin@dfci.harvard.edu only kill the cancer cell while sparing the normal cell. In mammalian cells, large-scale high throughput genomic screens for synthetic lethal interactors have been facilitated by the discovery of RNA interference (RNAi). Although RNAi was originally shown to serve as an antiviral defense mechanism, recent advances in our understanding of RNAi have made it possible to silence genes of interest in a highly specific manner by introducing short, double stranded, RNAs of defined sequences into cells [4]. RNAi has emerged as a powerful tool for the identification and validation of new targets for the pharmacological treatment of diseases in a systematic and unbiased manner. For example, genome-wide RNAi screens have recently been used to identify genes that, when silenced, kill cells that harbor mutant RAS but not cells that lack this mutation $[5,6]$.

Activating mutations in the $R A S$ oncogene (KRAS, HRAS and NRAS) are frequent in human cancer. For example, KRAS mutations occur in $60 \%$ of pancreatic cancers, $32 \%$ of cancers of the large intestine and in $17 \%$ of lung cancers [7]. RAS family members signal through numerous effector molecules with diverse functions. Prominent among these effectors are the RAF/ MAPK, PI3K and RAL proteins. The RAS proteins regulate critical processes in normal cells such as cell growth, motility, adhesion, and differentiation, to name but a few. So far, however, RAS itself has proven impossible to inhibit with drug-like small organic molecules. Moreover, there are theoretical concerns regarding possible toxicities that might emerge by inhibiting such a multifunctional signaling molecule.

The work of two groups in the November 2009 issue of Nature provides evidence that targeting the NF- $\mathrm{kB}$ signaling pathway might be one strategy to treat KRAS mutant tumors. Barbie et al. identified upstream regulators of the NF- $\mathrm{\kappa B}$ signaling pathway as being essential to the survival of cell lines that were dependent on oncogenic KRAS expression [8]. Meylan et al. showed that inhibition of the NF- $\mathrm{BB}$ pathway in an oncogenic KRAS mouse model of lung adenocarcinoma resulted in reduced tumor progression in vivo [9].

Hahn and coworkers inhibited genes thought to be important for cancer development and identified critical components of the KRAS signaling pathway that are essential only to the survival of cells that express mutant oncogenic KRAS [8]. Suppression of TBK1 by RNAi, like suppression of $K R A S$ itself, killed cancer cells that harbored oncogenic KRAS but not normal KRAS. TBK1, a member of the IкB kinase family, phosphorylates and degrades IкB, a potent inhibitor of NF- $\kappa \mathrm{B}$, thereby allowing the activation of the latter. NF$\kappa \mathrm{B}$ is a pleiotropic transcription factor that plays a key role in several physiological processes, most notably in cell proliferation, suppression of cell death, 
and inflammation. It has now become very clear that aberrant regulation of NF- $\kappa B$ contributes not only to cancer development, but also to chemoresistance and radioresistance [10].

To determine how TBK 1 contributes to survival in oncogenic $K R A S$ cells, the authors performed gene expression profiling in cancer cell lines and patient-derived tumors. They found that mutant KRAS tumors exhibited gene expression signatures indicative of NF- $\kappa \mathrm{B}$ activation, in addition to RAS activation, suggesting that tumors driven by mutant KRAS might depend on TBK1 and NF-kB for survival and that inhibiting TBK1 might provide a therapeutic advantage. Furthermore, the authors also found that suppression of TBK 1 resulted in downregulation of NF-кB-responsive anti-apoptotic proteins such as BCL-XL, c-Rel and p105 in $K R A S$ mutant cancer cells. Although TBK1 activates the inflammatory response through the regulation of IRF3 and IRF7, the authors did not see any evidence that this pathway was affected in the setting of oncogenic $R A S$.

The new findings of Hahn and coworkers described above are consistent with an earlier study showing that activation of NF- $\mathrm{\kappa B}$ is required for the survival of RAS transformed cells in vitro [11], as well as with a recent study linking TBK1 to RAS-induced oncogenic transformation. In the latter study TBK1 was shown to be activated by RALB, a downstream effector of RAS function, leading to diminished apoptosis in cancer cells [12].

Although synthetic lethal screens offer great promise, targets identified in vitro need to be validated in vivo in order to address the following questions: how does the synthetic lethal relationship function in the context of the tumor microenvironment and, secondly, do normal cells from other cell lineages depend on the target for their typical activity [1]? Indeed, given the magnitude of the role of NF- $\mathrm{\kappa B}$ in inflammation, standard tissue culture experiments might be insufficient to study its true function and the interaction of NF- $\mathrm{kB}$ dependent activities with, for example, the tumor microenvironment. This problem has now been partly addressed by Meylan and colleagues who engineered a mouse model of lung adenocarcinoma with activating oncogenic mutations in $K R A S$ [9]. Inactivating mutations in the tumor suppressor $p 53$ occurs in $50 \%$ of lung adenocarcinomas [13]. KRAS and p53 have opposing effects on NF- $\mathrm{KB}$ activity, RAS activates and $\mathrm{p} 53$ antagonizes this signaling network. Meylan et al. modeled lung adenocarcinoma in a mouse with concomitant loss of p53 and expression of oncogenic KRAS, which resulted in NF- $\kappa B$ activation. These mice developed advanced lung cancers that closely mimic the development of human lung adenocarcinomas. The authors further wanted to evaluate the role of NF- $\mathrm{KB}$ in tumor initiation and development. Mice were injected intratracheally with a virus encoding a hyperactive form of the IKB protein, which inhibits NF- $\kappa B$. These mice developed significantly less tumors than mice expressing a control gene. Inhibition of $\mathrm{NF}-\kappa \mathrm{B}$ at the time of tumor initiation had a profound effect on subsequent tumor development. Furthermore, inhibition of NF- $\mathrm{\kappa B}$ in established tumors led to slowing of tumor growth, although this did not lead to their complete regression.

The work of Barbie et al. and Meylan et al. underscore the importance of the NF- $\mathrm{KB}$ signaling network for the survival of KRAS mutant cells in vitro. One of the major challenges in treating cancers characterized by increased NF$\kappa \mathrm{B}$ activity is the pleiotropic activities of this transcription factor in tumor maintenance, involving both cell-intrinsic and cell-extrinsic effects, and in normal host physiology. Given its vital role in the inflammatory and innate immune response, prolonged suppression of $\mathrm{NF}-\kappa \mathrm{B}$ activity might ultimately cause side effects. Intermittent administration of an NF- $\mathrm{kB}$ antagonist, however, might theoretically suffice to induce apoptosis in KRAS mutant tumors without causing immunosuppression. Moreover, targeting individual nodes in the NF$\kappa \mathrm{B}$ network, rather than NF- $\kappa \mathrm{B}$ itself, might help to limit toxicity. It is also worth noting that Bortezomib, which has been suggested to inhibit NF- $\mathrm{kB}$ by stabilizing I $\mathrm{KB}$ (among a host of other activities), induces responses in multiple myeloma at tolerable doses [14].

Targeting tumor cells while preserving the integrity of normal cells is the 'holy grail' in the fight against cancer. Identifying genes that behave in a synthetic lethal manner is theoretically one way to achieve this objective. Recent clinical trials of PARP inhibitors in patients with tumors characterized by defects in homologous recombination suggest that this paradigm can, indeed, lead to drugs with enhanced efficacy and safety profiles [15]. The work of Barbie et al. and of Meylan et al., together with previously published studies $[5,6]$, offer hope that it will soon be possible to pharmacologically attack RAS-driven tumors.

\section{References}

1 Kaelin WG, Jr Synthetic lethality: a framework for the development of wiser cancer therapeutics. Genome Med 2009; 1:99.

2 Hartwell LH, Szankasi P, Roberts CJ, Murray AW, Friend SH. Integrating genetic approaches into the discovery of anticancer drugs. Science 1997; 278:1064-1068.

3 Kaelin WG, Jr The concept of synthetic lethality in the context of anticancer therapy. Nat Rev Cancer 2005; 5:689698.

4 Elbashir SM, Harborth J, Lendeckel W, Yalcin A, Weber K, Tuschl T. Duplexes of 21-nucleotide RNAs mediate RNA interference in cultured mammalian cells. Nature 2001; 411:494-498.

5 Luo J, Emanuele MJ, Li D, et al. A genome-wide RNAi screen identifies multiple synthetic lethal interactions with the Ras oncogene. Cell 2009; 137:835-848.

6 Scholl C, Frohling S, Dunn IF, et al. 
Synthetic lethal interaction between oncogenic KRAS dependency and STK33 suppression in human cancer cells. Cell 2009; 137:821-834.

7 Karnoub AE, Weinberg RA. Ras oncogenes: split personalities. Nat Rev Mol Cell Biol 2008; 9:517-531.

8 Barbie DA, Tamayo P, Boehm JS, et al. Systematic RNA interference reveals that oncogenic KRAS-driven cancers require TBK1. Nature 2009; 462:108112.

9 Meylan E, Dooley AL, Feldser DM, et al. Requirement for NF- $\kappa \mathrm{B}$ signalling in a mouse model of lung adenocarcinoma. Nature 2009; 462:104-107.

10 Baud V, Karin M. Is NF- $\kappa$ B a good target for cancer therapy? Hopes and pitfalls. Nat Rev Drug Discov 2009; 8:33-40.

11 Mayo MW, Norris JL, Baldwin AS. Ras regulation of NF-kappa B and apoptosis. Methods Enzymol 2001; 333:7387.

12 Chien Y, White MA. Characterization of RalB-Sec5-TBK1 function in human oncogenesis. Methods Enzymol 2008;
438:321-329.

13 Herbst RS, Heymach JV, Lippman SM. Lung cancer. N Engl J Med 2008; 359:1367-1380.

14 Hideshima T, Ikeda H, Chauhan D, et al. Bortezomib induces canonical nuclear factor- $\mathrm{kB}$ activation in multiple myeloma cells. Blood 2009; 114:10461052.

15 Fong PC, Boss DS, Yap TA, et al. Inhibition of poly(ADP-ribose) polymerase in tumors from BRCA mutation carriers. N Engl J Med 2009; 361:123-134. 